\title{
Elaboración de escalas de valoración de obstáculos y medidas para abordar la violencia de género en las escuelas
}

\author{
Development of scales to study barriers and needed measures
}

to address gender-based violence in schools

\author{
Lorena Saletti-Cuesta ${ }^{1}$, Lila Aizenberg ${ }^{2}$, Eduardo Torres ${ }^{3}$ \\ y Lucía Florencia Sánchez ${ }^{4}$
}

${ }^{1}$ Doctora en Salud, Antropología e Historia. Investigadora Adjunta en el Centro de Investigación y Estudios sobre Cultura y Sociedad - Consejo Nacional de Investigaciones Científicas y Técnicas (CIECS - CONICET) - Universidad Nacional de Córdoba (UNC). Docente del

Doctorado de Ciencias de la Salud (Facultad de Ciencias Médicas - UNC).

E-mail: lorenasaletti@unc.edu.ar

${ }^{2} \mathrm{PhD}$. en Sociología. Investigadora Adjunta en el Centro de Investigación y Estudios sobre Cultura y Sociedad (CIECS - CONICET - Universidad Nacional de Córdoba).

E-mail: lila.aizenberg@gmail.com

${ }^{3}$ Doctor en Demografía. Investigador Adjunto en el Centro de Investigación y Estudios sobre Cultura y Sociedad (CIECS - CONICET - Universidad Nacional de Córdoba). Profesor Auxiliar en la materia Análisis Demográfico y en el Departamento de Estadística y Matemática (Facultad de Ciencias Económicas, UNC). Docente de la Maestría en Demografía (Centro de Estudios

Avanzados, UNC) y del Doctorado en Demografía. E-mail: torresedu@gmail.com ${ }^{4}$ Licenciada en Psicología. Becaria doctoral del Consejo Nacional de Investigaciones Científicas y Técnicas, en el Centro de Investigación y Estudios sobre Cultura y Sociedad (CIECS CONICET - Universidad Nacional de Córdoba). Docente de la Universidad Provincial de Córdoba. Estudiante del Doctorado en Psicología en la Universidad Nacional de Córdoba. E-mail: luciasanchez09@gmail.com

El trabajo que se presenta se enmarca en un asesoramiento científico técnico realizado para el Programa Escuelas Libres de Violencias de género del Ministerio de Justicia

y Derechos Humanos de la Provincia de Córdoba.

Los autores agradecen a todas las personas que participaron cumplimentando el cuestionario

y especialmente, la colaboración de las compañeras del Programa Escuelas Libres de

Violencias de Género del Ministerio de Justicia y Derechos Humanos de la Provincia de Córdoba.

Centro de Investigación y Estudios sobre Cultura y Sociedad (CIECS - CONICET - UNC). Córdoba, Argentina.

\section{Resumen}

El personal docente cumple un rol clave en la prevención de la violencia de género (VG) en el ámbito educativo. Este trabajo tiene como objetivo validar al contexto local dos escalas para indagar cómo el personal docente y directivo valoran los obstáculos para abordar 
la VG y conocer su valoración respecto a las medidas propuestas para mejorar la prevención y la forma de abordarla. Se realizó un estudio descriptivo, transversal y multicéntrico con personal docente y directivo de escuelas públicas de Córdoba, Argentina. Para ello, se utilizó un cuestionario autoadministrado que incluyó dos escalas, una sobre obstáculos y otra sobre medidas. Se realizó análisis descriptivo, bivariante, factorial exploratorio y alfa de Cronbach y se construyó un modelo de regresión múltiple utilizando cada factor como variable dependiente. La escala validada de obstáculos se compone de diez preguntas y la escala de medidas incluye trece. Los resultados de regresión múltiple indican que los vínculos y prejuicios funcionan como obstáculos importantes para el nivel primario. La importancia otorgada a la ayuda especializada, como medida para prevenir la VG, se asocia a los equipos educativos que han manifestado necesidad de formación en este tema. Quienes han realizado actividades de prevención en las escuelas de nivel primario valoran más la transversalización de la perspectiva de género como medida. Las escuelas con personal en situación de VG consideran la formación en VG como medida de gran relevancia. Las escalas validadas en este estudio son fiables y recogen dimensiones empíricas de las valoraciones sobre obstáculos y medidas en relación con el abordaje de la VG en las escuelas.

Palabras clave: escala, sexismo, violencia de género, escuela, estudio cuantitativo, Argentina.

\section{Abstract}

Teachers play a key role in the prevention of gender violence (GBV) in the educational field. However, studies evaluating the opinions of teaching and management staff regarding the necessary measures to prevent GBV or the obstacles they must face when addressing this problem in the educational field in our context are incipient. This work aims to validate two scales to know the opinions and evaluations of the educational personnel on the obstacles and the measures they consider necessary to tackle and prevent GBV in schools. A descriptive, cross-sectional and multicenter study was carried out to an intentional non-probability sampling. The study population was the directing and teaching staff of schools in the Province of Córdoba, Argentina. The study variables were: sex, years of experience in the position, age, educational level of the center (initial / primary / middle / tertiary), area (urban / rural), locality where the faceto-face training was carried out, prevention activities carried out in the educational center focused on: students, families, and the educational team; GBV training activities carried out by the educational team, women and/or families identified in GBV situation, families with judicial restraining order, team interventions education before GBV of the students and/or families, staff of the educational team that has lived or is experiencing a situation of GBV, meetings of the educational team with local community organizations to articulate activity to prevent and/or address GBV, need for training on GBV manifested by the teaching team. To measure opinions on the obstacles and measures necessary to address GBV in schools, we applied a self-administered questionnaire with two scales, one on obstacles and the other on measures, based on Spanish scales. A descriptive, bivariate analysis of the relationship of all variables with sex, exploratory factor and Cronbach's alpha was performed. Finally, a multiple regression model was constructed to test the convergent validity using each factor as the dependent variable. The first scale of ten items identifies a dimension of obstacles related to family ties and existing prejudices in the educational community. The second dimension, values aspects related to the scarcity of resources. For its part, the thirteen item scale of measurements has three dimensions. The first dimension, groups measures related to specialized help to address GBV, such as prevention programs, materials, and coordination with external organizations. The second groups the measures related to the need to 
have gender mainstreaming measures in the approach to GBV at school. The third dimension reflects the importance of staff training on these topics. Bonds and biases function as major obstacles for the elementary level. The importance given to specialized help, as a measure to prevent GBV, is mainly associated with educational teams that have expressed a need for training in this area. Those who have carried out prevention activities and primary schools, value more the mainstreaming of the gender perspective as a measure. Schools with staff who have lived or are experiencing a GBV situation consider GBV training as a highly relevant measure. The scales validated in this study are reliable and include empirical dimensions of the assessments of obstacles and measures in relation to the GBV approach in schools.

Keywords: scales, gender-based, violence, school, quantitative study, Argentina.

\section{Introducción}

Por su importancia y sus consecuencias, la violencia de género (VG) es una problemática mundial que impide en el ámbito escolar, especialmente a las niñas, ejercer su derecho a la educación segura, integradora y de calidad (Flores Bernal, 2005; Global Education Monitoring Report Team y United Nations Girls' Education Initiative, 2015; Trucco e Inostroza, 2017). Además, la VG puede acarrear un mayor ausentismo escolar, malos resultados académicos, deserción escolar, baja autoestima, depresión, embarazos e infecciones de transmisión sexual como el VIH, todo lo cual tiene un efecto perjudicial sobre el aprendizaje y el bienestar (Global Education Monitoring Report Team y United Nations Girls' Education Initiative, 2015).

En relación con las escuelas, la VG es definida como los actos o las amenazas de violencia sexual, física o psicológica que suceden dentro y fuera de la escuela, perpetrados hacia integrantes de la comunidad educativa como resultado de las desigualdades de género y de poder (Aizenberg, Saletti-Cuesta y Torres, 2019; Global Education Monitoring Report Team y United Nations Girls' Education Initiative, 2015). Las distintas formas de VG en la escuela se superponen y se refuerzan mutuamente. Estas formas o tipos de VG pueden estar asociados a la reproducción de normas y estereotipos de género, es decir a una violencia simbólica por ejemplo, así como también a amenazas explícitas o actos de violencia física, bullying, acoso verbal o sexual, coerción y agresión sexual o violación (Global Education Monitoring Report Team y United Nations Girls' Education Initiative, 2015). En ese sentido, lo que ocurre en las escuelas no es ajeno a cómo la VG se manifiesta en otros ámbitos de la sociedad. Así, si bien es útil diferenciar y distinguir los diversos tipos de violencia, según la Ley Nacional N. ${ }^{\circ}$ 26.485 de protección integral para prevenir, sancionar y erradicar la violencia contra las mujeres en Argentina (Senado y Cámara de Diputados de la Nación Argentina, 2009), los tipos de violencia establecidos son: psicológica, sexual, económica y patrimonial, simbólica, física y política. En la práctica, esta diferenciación resulta artificial porque, en general, los tipos de VG suelen ocurrir de forma simultánea o superpuesta (Krebs, Breiding, Browne y Warner, 2011).

Las instituciones educativas han sido señaladas como un prisma que refracta la cultura patriarcal de las sociedades (Flores Bernal, 2005; Míguez, 2009; Ruiz-Ramírez y Ayala-Carillo, 2016) y la VG como un problema de relevancia que atraviesa la vida cotidiana de las escuelas (Kornblit, 2008; Pinedo González, Arroyo González y Berzosa Ramos, 2018).

Es reconocido que las escuelas tienen una importancia trascendente en la construcción de subjetividades y que las experiencias personales de las y los docentes (a partir de ahora, "los docentes"), como su propia experiencia escolar, marcan sus prácticas cotidianas en el aula, lo que facilita u obstaculiza el tratamiento de ciertas temáticas (Kornblit 
et al., 2014) como podría ser la VG. La literatura nacional e internacional ha reconocido distintos mecanismos que conducen a la reproducción de la VG en el ámbito escolar. Se destaca entre ellos la escasez de recursos destinados a la ejecución de los programas (Díaz Aguado, 2009); la implementación errática de los marcos normativos sobre prevención de VG en el ámbito educativo; la dificultad para su abordaje en las aulas (Barredo Ibáñez et al, 2014; Morgade, 2009) y la escasez de asignaturas específicas y/o transversales que permitan reflexionar sobre la naturalización de las diferencias de género (Moreno, 2000). También se ha señalado que, si bien los docentes reconocen cambios en sus miradas respecto al género, aún persisten resistencias vinculadas a sus propias trayectorias o biografías que obstaculizan el desarrollo de estrategias educativas para la igualdad de género, en general, y para la educación sexual integral, en particular (Faur, Gogna y Binstock, 2015). Se ha indicado que persisten importantes dificultades para identificar tanto las desigualdades de género y relaciones de poder producto de ellas, como al machismo limitante en el desarrollo de una plena ciudadanía, lo que permitiría la posibilidad de salirse de cánones heteronormativos por parte de los alumnos, las alumnas y los docentes (Flores Bernal, 2005; Kornblit, Sustas y Di Leo, 2014).

Así como a los espacios educativos se los ha señalado como ámbitos de re/producción de los procesos de socialización de desigualdades, también se los ha identificado como agentes clave en la prevención de violencias e inequidades basadas en el género (Alonso Hernández, Cacho Sáez, González Ramos, Herrera Álvarez y Ramírez García, 2016; Díaz-Aguado, 2002, 2006; Díaz-Aguado y Carvajal, 2011; Flores Bernal 2005; Moriana Mateo, 2017; Pinedo González, Arroyo González y Berzosa Ramos, 2018; Ruiz Repullo, 2017). El fomento de las relaciones cooperativas e igualitarias entre mujeres y hombres y la implementación de un currículum explícito que eduque en la no-vio- lencia han sido destacadas como estrategias para prevenir la VG desde las escuelas (DíazAguado, 2006; Ruiz Repullo, 2017). Para ello, mayoritariamente, se han elaborado y distribuido materiales y, en menor medida, implementado programas puntuales sobre coeducación y fomento de la igualdad (Díaz-Aguado, 2006). Los programas orientados al fomento de la igualdad han demostrado efectividad en la disminución del sexismo, del acoso entre pares, tanto dentro como fuera de la escuela, y la mejora de las relaciones con el profesorado (Díaz-Aguado, 2006).

Asimismo, distintos trabajos han señalado el rol protagónico que tienen los docentes en la prevención y el abordaje de VG en el ámbito educativo, ya que la formación en perspectiva de género a profesionales es un aspecto clave para alcanzar la equidad dentro del aula (Bonder, 2010; Morgade, 2009; Morgade, 2011; Pinedo González, Arroyo González y Berzosa Ramos, 2018; Ruiz Repullo, 2017). En ese sentido, se ha señalado que la transformación de las estructuras patriarcales en la escuela no depende solo de la formación reglada del profesorado, sino de la posibilidad de que los docentes puedan reflexionar sobre su propio rol como agentes transmisores de valores y conocimientos tradicionales de género, de las relaciones de poder entre los sexos y de las formas de disciplinar a los cuerpos, entre otras cuestiones de la práctica educativa (Bonder, 2010; Morgade y Alonso, 2008; Tomasini, 2010; Torres San Miguel, 2010).

Desde la década pasada, Argentina es uno de los países pioneros en la sanción de leyes vinculadas a la prevención de las VG. Tanto la Ley de Educación Sexual Integral (Ley 26.150) del año 2006 como la Ley de protección integral para prevenir, sancionar y erradicar la violencia contra las mujeres en los ámbitos en que desarrollen sus relaciones interpersonales (Ley 26.485) del año 2009 constituyen el marco normativo que sitúa a las escuelas, junto a otros sectores de la sociedad, como agentes fundamentales de prevención y abor- 
daje de la VG. En esta línea, el Plan Nacional de Acción contra las violencias por motivo de género (Ministerio de las Mujeres, Géneros y Diversidad, 2020) reconoce a la educación como uno de los ámbitos estratégicos para la prevención de la VG, y define para este sector distintas líneas de acción, que van desde la revisión con perspectiva de género de los planes de formación docente y de la currícula de los distintos niveles, como la implementación de campañas para la deconstrucción de los estereotipos de género, hasta acciones de sensibilización y capacitación sobre las distintas VG, incluyendo las cibernéticas. A su vez, provincias como Córdoba han ejecutado programas específicos para fortalecer la prevención de las VG desde las escuelas. El Programa Escuelas Libres de Violencias de Género (https://www.cba.gov.ar/escuelas-libres-de-violencia/), implementado desde 2016 por el Ministerio de Justicia y Derechos Humanos y el Ministerio de Educación del Gobierno de la Provincia de Córdoba, busca propiciar y profundizar procesos de sensibilización y capacitación en el sistema educativo formal a fin de promover la transformación de patrones socioculturales que naturalizan la VG, a través de capacitaciones a personal docente y directivo y a futuros profesionales de la educación.

La validación de escalas sobre medidas y obstáculos para el abordaje de la VG en la población educativa en Argentina cobra particular relevancia, especialmente a la luz los estudios que ya han señalado la existencia de una política pública errática en el ámbito educativo (Bonder, 2010), así como de las resistencias de los docentes respecto a la implementación de programas destinados al abordaje de la educación sexual integral (Faur, Gogna y Binstock, 2015). Asimismo, la identificación de obstáculos y medidas - a partir de escalas- resulta particularmente enriquecedora para complementar el campo de estudio de la VG y la educación sexual integral en el ámbito educativo en Argentina, el cual ha estado principalmente orientado a identificar, desde abordajes cualitativos, las representaciones sociales y experiencias en la implementación de contenidos y programas (Faur, Gogna y Binstock, 2015; Tomasini, 2010; Wainerman, Di Virgilio y Chami, 2008).

El objetivo de este trabajo es validar dos escalas para el contexto local: la primera, diseñada para indagar cómo valoran los obstáculos para abordar la VG los equipos educativos. La segunda escala pretende estudiar la valoración de distintas medidas propuestas para mejorar la prevención y el abordaje de la VG. Estas escalas forman parte de un cuestionario más amplio, diseñado para monitorear el impacto del Programa Provincial Escuelas Libres de Violencias de Género de la Provincia de Córdoba en el año 2017, que incluyó otras escalas como la utilizada para medir opiniones sobre VG (Aizenberg, Saletti-Cuesta y Torres, 2019) y otros indicadores para conocer la prevalencia de situaciones de VG en las escuelas.

\section{Método}

Se realizó un estudio descriptivo, transversal y multicéntrico.

\section{Participantes}

La población de estudio fue el personal directivo y docente de 528 escuelas de la provincia de Córdoba, Argentina. El criterio de inclusión fue asistir a la primera capacitación presencial organizada en 2017 por el Programa Escuelas libres de VG. Se realizó un muestreo intencional no probabilístico. La cantidad total de participantes que contestó la encuesta fue de 499.

\section{Procedimiento}

Antes del inicio de la primera capacitación presencial y durante la inscripción y presentación de la capacitación, se distribuyó en manoel cuestionario a los participantes. Se les explicó que el cuestionario era autocumplimentado, 
que formaba parte del monitoreo del Programa Escuelas Libres de VG y que el tratamiento de los datos era confidencial y anónimo, y se solicitó su consentimiento informado para formar parte del estudio. Si bien el estudio no contó con la aprobación de un comité de ética, fue autorizado por el Ministerio de Educación y el Ministerio de Justicia y Derechos Humanos de la provincia de Córdoba. Durante todo el proceso se resguardaron todos los derechos de las personas participantes y se adhirió a los valores y principios éticos vigentes. La participación fue voluntaria y las personas fueron libres de decidir si participar o no y de abandonar en cualquier momento sin tener que dar explicaciones. Quienes decidieron participar, autocumplimentaron y entregaron en mano el cuestionario antes de comenzar la capacitación. Por otro lado, algunas personas lo entregaron al finalizar la capacitación.

\section{Instrumento}

Para medir las opiniones sobre los obstáculos y las medidas necesarias para abordar la VG en las escuelas, se diseñó un cuestionario autoadministrado que incluyó, además de otras variables, dos escalas, una sobre obstáculos y otra sobre medidas. Se entendió como obstáculo toda barrera que dificulta el abordaje integral de las VG en las escuelas y el avance hacia la igualdad y la prevención de las VG desde la educación, y se entendió como medidas las herramientas para visibilizar, prevenir y erradicar las VG desde la educación (Díaz Aguado y Carvajal Gómez, 2011). Para adaptar y validar este contexto en dos escalas, se realizó un juicio de expertas para valorar la comprensión y claridad, modificar la redacción de ítems que así lo requerían e incorporar elementos relevantes en ese contexto. Las expertas fueron seleccionadas por su experiencia y conocimientos en el tema.

La escala de obstáculos se compuso de diez ítems. Las preguntas 1 y 3 fueron de elaboración propia, ya que lo que interesaba era profundizar en las relaciones y vínculos como elemento para abordar las VG, tal como es señalado por Díaz Aguado y Carvajal Gómez (2011). El resto de las preguntas fueron extraídas y adaptadas de la escala sobre obstáculos elaborada por Díaz Aguado y Carvajal Gómez (2011). De dicha escala, se seleccionaron los ítems pertinentes y adaptables al contexto que se quería analizar, excluyendo por ejemplo "El sexismo existente en los libros de texto", puesto que no todas las escuelas en la provincia de Córdoba utilizan libros de texto como fue señalado en el juicio de expertas. La confiabilidad de la escala original es explicada por tres factores: (1) falta de implicación del profesorado y sexismo escolar, con $\alpha=.79$ (IC del $95 \%$ : .78 - .80); (2) falta de implicación y sexismo en las familias y en los medios de comunicación, con $\alpha=.76$ (IC del $95 \%$ : .74 - .78); y (3) obstáculos derivados de la organización escolar, con $\alpha=.84$ (IC del $95 \%: .83$ - .85) (Díaz Aguado y Carvajal Gómez, 2011).

Respecto a la escala de medidas, las preguntas 4, 5, 6 y 7 fueron de elaboración propia, ya que interesó evaluar las propuestas que el Programa Escuelas Libres de Violencia presentaba para el contexto en estudio. El resto de las preguntas fueron extraídas de la escala de valoración de posibles medidas para mejorar la igualdad y la prevención de la VG desde el centro educativo, elaborada por Díaz Aguado y Carvajal Gómez (2011) y adaptada al contexto escolar mediante juicio de expertas. El único ítem no incluido de dicha escala fue "Mejora del Plan de Acción Tutorial para incrementar su impacto sobre estos temas", puesto que no existe dicha estrategia en las escuelas de Córdoba. No se encuentra publicada la confiabilidad de la escala original. La escala de respuesta de ambas escalas fue tipo Likert con cuatro opciones: nada, poco, bastante, mucho.

Otras variables de estudio incluidas en el cuestionario fueron: sexo, años de experiencia en el cargo, edad, nivel educativo del centro (inicial/primario/medio/terciario), zona (urbana/rural), localidad donde se realizó la formación presencial, actividades de preven- 
ción realizadas en el centro educativo dirigidas al alumnado ( $s i$ / no), a las familias ( $s i$ / no) y al equipo educativo ( $\mathrm{si} / \mathrm{no}$ ), actividades de formación sobre $\mathrm{VG}$ realizadas por el equipo educativo ( $s i$ / no), mujeres y/o familias identificadas en situación de VG (sí / no), familias con orden de restricción judicial (es decir, medidas de protección ordenadas por la justicia luego de una denuncia para disminuir el riesgo) (sí / no), intervenciones del equipo educativo ante VG del alumnado y/o las familias (si / no), personal del equipo educativo que ha vivido o vive una situación de VG (sí / no), reuniones del equipo educativo con organizaciones locales de la comunidad para articular actividad para prevenir y/o abordar VG (si / no), necesidad de formación sobre VG manifestada por el equipo docente ( $s i / n o$ ).

\section{Análisis de datos}

Se realizó un análisis descriptivo, bivariante de la relación de todas las variables con el sexo y un análisis factorial exploratorio para analizar la validez de constructo, es decir descubrir la estructura interna de un número de variables creando nuevos factores que acumulan la varianza común y que poseen significado interpretativo. Siguiendo las recomendaciones de Costello y Osborne (2005), se analizaron las saturaciones factoriales de los ítems y se retuvieron aquellos con una saturación factorial mayor a .35 , que el ítem no tuviera una saturación superior en otro factor y que, además, tuvieran coherencia interna. Asimismo, se estimó el alfa de Cronbach para evaluar la confiabilidad, consistencia interna y fiabilidad de cada escala. Finalmente, se construyó un modelo de regresión múltiple para probar la validez convergente, utilizando cada factor como variable dependiente y las 26 incluidas en las escalas fueron las variables independientes. Se utilizó SPSS v.19.

\section{Resultados}

La tasa de respuesta al cuestionario fue de $72 \%(n=499)$, el $90 \%$ fueron mujeres $(\mathrm{n}=432)$. La edad media fue de 47.5 años (mujeres: 47.59 años; varones: 47.17 años). La media de años de experiencia en el cargo fue de 8.91 (mujeres: 9.2 años; varones 6.6 años).

Respecto al nivel educativo de las escuelas, el $25 \%$ eran de nivel inicial, $43.2 \%$ de primario, $31.6 \%$ de secundario y solo el .2\% de terciario. El $72.2 \%$ pertenecían a escuelas de zonas urbanas y el $27.8 \%$ al rural. En el $54.6 \%$ de los casos, el equipo docente había planteado la necesidad de formarse en temas vinculados a la VG. El 49.4 \% participó en alguna reunión con la comunidad para articular actividades de prevención relacionadas con la VG. El 72.1\% de las escuelas realizó alguna actividad de prevención de la VG dirigida al alumnado, el $70 \%$ dirigidas a las familias, y el $56.9 \%$ al equipo educativo. El $51.1 \%$ indicó que el equipo educativo realizó actividades de formación sobre VG. El 26.7 \% respondió que el equipo educativo realizó alguna intervención ante VG del alumnado y/o las familias. En relación con la detección de casos de $\mathrm{VG}$, el $51.1 \%$ indicó que hay mujeres/familias identificadas en la escuela que viven o han vivido una situación de $\mathrm{VG}$; el $40.1 \%$, que hay familias con orden de restricción judicial vigente (por ejemplo, botón antipánico), y el $20.8 \%$ respondió que hay personal del equipo educativo que ha vivido o vive una situación de VG.

El análisis factorial exploratorio de la escala de obstáculos identificó dos dimensiones que explicaron el $54.04 \%$ de la varianza. El factorial de la escala de medidas identificó tres dimensiones que explicaron el $67.50 \%$ de la varianza. La escala final de obstáculos se compone de diez preguntas y la escala de medidas incluye trece. Las cargas factoriales y el alfa de Cronbach de los cinco factores aparecen en la tabla 1. 
Tabla 1

Cargas factoriales y alfa de Cronbach de los dos factores de la escala de obstáculos y de los tres factores de la escala de medidas.

\begin{tabular}{|c|c|c|c|c|c|}
\hline Ítems / Factores & F1 & $\mathbf{F} 2$ & F3 & F4 & F5 \\
\hline Mala calidad de relaciones entre alumnas y alumnos & .701 & & & & \\
\hline $\begin{array}{l}\text { Dificultad de contrarrestar desde la escuela la influencia de los } \\
\text { medios de comunicación sobre este tema }\end{array}$ & .547 & & & & \\
\hline Relaciones conflictivas entre equipo docente y familias & .746 & & & & \\
\hline El sexismo existente en parte del profesorado & .611 & & & & \\
\hline $\begin{array}{l}\text { Rechazo del profesorado a trabajar en este tema, porque cree } \\
\text { que no es su función }\end{array}$ & .728 & & & & \\
\hline La falta de formación del profesorado para tratar estos temas & & .697 & & & \\
\hline $\begin{array}{l}\text { La insuficiencia de los recursos con los que dispone la escuela } \\
\text { para afrontar este reto }\end{array}$ & & .726 & & & \\
\hline $\begin{array}{c}\text { Falta de tiempo para dar todo el temario y tratar estos temas no } \\
\text { incluidos en las asignaturas }\end{array}$ & & .791 & & & \\
\hline $\begin{array}{l}\text { Tendencia a tratar estos temas en una fecha determinada, sin } \\
\text { incorporarlos de forma sistemática al currículo }\end{array}$ & & .737 & & & \\
\hline El sexismo existente en la educación familiar & & .534 & & & \\
\hline $\begin{array}{l}\text { Disponer de ayuda especializada en este tema que ayude a } \\
\text { incluirlo en el centro }\end{array}$ & & & .472 & & \\
\hline $\begin{array}{c}\text { Mayor cooperación con los organismos encargados de trabajar } \\
\text { sobre estos temas }\end{array}$ & & & .637 & & \\
\hline $\begin{array}{l}\text { Disponer de materiales que faciliten el tratamiento de estos } \\
\text { temas en las aulas }\end{array}$ & & & .619 & & \\
\hline $\begin{array}{l}\text { Mayor implicación de otros programas del Ministerio de } \\
\text { Educación en el seguimiento de este tema }\end{array}$ & & & .685 & & \\
\hline $\begin{array}{l}\text { Implantación de programa específico para prevenir la VG en las } \\
\text { aulas }\end{array}$ & & & .681 & & \\
\hline $\begin{array}{l}\text { Disponer de recursos profesionales (ej. Gabinete } \\
\text { psicopedagógico) en el centro para abordar la VG }\end{array}$ & & & .838 & & \\
\hline $\begin{array}{l}\text { Disponer de un protocolo de actuación para saber cómo actuar } \\
\text { en caso de conocer VG entre el alumnado o en sus familias }\end{array}$ & & & .794 & & \\
\hline $\begin{array}{l}\text { Implantación o mejora del Acuerdo Escolar de Convivencia, } \\
\text { incluyendo estos temas de forma sistemática y generalizada }\end{array}$ & & & & 609 & \\
\hline $\begin{array}{c}\text { Incluir estos temas, como un más, en los programas de } \\
\text { asignaturas evaluables }\end{array}$ & & & & .731 & \\
\hline $\begin{array}{c}\text { Mayor implicación de Inspección en el seguimiento de este } \\
\text { tema }\end{array}$ & & & & .656 & \\
\hline
\end{tabular}




\begin{tabular}{c|c|c|c|c|c} 
Ítems / Factores & F1 & F2 & F3 & F4 & F5 \\
\hline $\begin{array}{c}\text { Formación especializada sobre prevención de la VG } \\
\begin{array}{c}\text { Formación sobre cómo incluir estos temas en las asignaturas } \\
\text { de forma que no vayan en detrimento de los temas que deben } \\
\text { tratarse }\end{array}\end{array}$ & & & & & .801 \\
$\begin{array}{c}\text { Formación especializada sobre prevención de la violencia desde } \\
\text { una perspectiva integral, que incluya la VG y otras violencias } \\
\text { Alfa de Cronbach }\end{array}$ & & & & & \\
\hline
\end{tabular}

Nota: F1: Escala obstáculos: factor vínculos y prejuicios; F2: Escala obstáculos: factor escasez de recursos; F3: Escala medidas: factor ayuda especializada; F4: Escala medidas: factor transversalización de la perspectiva de género; F5: Escala medidas: factor formación en VG.

En la escala de obstáculos, al primer factor se lo llamó Vínculos y prejuicios e integra cinco preguntas que identifican agentes y relaciones que influyen en la prevención y el abordaje de la VG en la escuela. El alfa de Cronbach de este factor fue .748. El segundo factor, Escasez de recursos, integra cinco preguntas sobre obstáculos comprendidos como factores que dependen, principalmente, del equipo docente $\mathrm{y} / \mathrm{o}$ de las escuelas, y se vinculan con elementos que dificultan la prevención y el abordaje de temas como las VG. El alfa de Cronbach de este factor fue .778. En cuanto a la confiabilidad, los alfa de Cronbach obtenidos en nuestra escala son similares a los de la escala original, por tanto, la inclusión de nuevos ítems no influyó en este aspecto.

Respecto a la escala de medidas, al primer factor lo se lo denominó Ayuda especializada e integra siete preguntas sobre medidas vinculadas a la ayuda y/o intervenciones especializadas para integrar la prevención de la VG en las escuelas. El alfa de Cronbach de este factor fue .862. El segundo factor, Transversalización de la perspectiva de género, integra tres preguntas que reflejan la integración transversal de medidas que incorporen la prevención de la VG. El alfa de Cronbach de este factor fue .624. El tercer factor es Formación en VG e integra tres preguntas vinculadas a la importancia de implementar estrategias destinadas al fortalecimiento de la formación de los equipos educativos en temas de VG y su alfa de Cronbach fue .877.

Las relaciones crudas y ajustadas de todas las variables estudiadas con cada factor de cada escala para el conjunto de la muestra se reflejan en las tablas 2 y 3 .

Tabla 2

Regresión lineal múltiple para el factor Vínculos y prejuicios y para el factor Escasez de recursos de la escala Obstáculos.

\begin{tabular}{c|c|c|c|c|c}
\hline Factor Vínculos y prejuicios \\
Variable & $\begin{array}{c}\text { Cat. de } \\
\text { referencia }\end{array}$ & $\begin{array}{c}\text { Cat. de } \\
\text { riesgo }\end{array}$ & Beta & IC 95 \% & P \\
\hline $\begin{array}{c}\text { Edad } \\
\begin{array}{c}\text { Actividades de prevención de la VG } \\
\text { realizadas en el centro dirigidas a las } \\
\text { familias }\end{array}\end{array}$ & No & Sí & -.349 & $-.619 ; .078$ & .012
\end{tabular}




\begin{tabular}{|c|c|c|c|c|c|}
\hline $\begin{array}{c}\text { Factor Vínculos y prejuicios } \\
\text { Variable }\end{array}$ & $\begin{array}{l}\text { Cat. de } \\
\text { referencia }\end{array}$ & $\begin{array}{l}\text { Cat. de } \\
\text { riesgo }\end{array}$ & Beta & IC $95 \%$ & $\mathbf{P}$ \\
\hline $\begin{array}{l}\text { Familias con órdenes de restricción } \\
\text { judicial }\end{array}$ & No & Sí & .323 & $.080 ; .566$ & .009 \\
\hline Nivel & $\begin{array}{l}\text { Otros } \\
\text { niveles }\end{array}$ & Primario & -.326 & $-.566 ;-.086$ & .008 \\
\hline Años de experiencia en el cargo & & & -.019 & $-.034 ;-.003$ & .021 \\
\hline $\begin{array}{l}\text { Equipo educativo ha planteado la } \\
\text { necesidad de formación sobre VG }\end{array}$ & No & Sí & -.277 & $-.515 ;-.040$ & .022 \\
\hline Localidad Deán Funes [DF] & No es de DF & DF & .335 & $-.050 ; .720$ & .088 \\
\hline Zona & Urbana & Rural & .202 & $-.060 ; .464$ & .131 \\
\hline $\begin{array}{c}\text { Factor Escasez de recursos } \\
\text { Variable }\end{array}$ & $\begin{array}{l}\text { Cat. de } \\
\text { referencia }\end{array}$ & $\begin{array}{l}\text { Cat. de } \\
\text { riesgo }\end{array}$ & Beta & IC $95 \%$ & $\mathbf{P}$ \\
\hline Zona & Urbana & Rural & .275 & $.034 ; .516$ & .026 \\
\hline Nivel & $\begin{array}{l}\text { Otros } \\
\text { niveles }\end{array}$ & Inicial & -.353 & $-.645 ;-.061$ & .018 \\
\hline Localidad DF & No es de DF & DF & .584 & $.218 ; .949$ & .002 \\
\hline $\begin{array}{l}\text { Personal del equipo educativo que ha } \\
\text { vivido o vive una situación de } \mathrm{VG}\end{array}$ & No & Sí & .271 & $.000 ; .543$ & .050 \\
\hline Nivel & $\begin{array}{l}\text { Otros } \\
\text { niveles }\end{array}$ & Medio & .316 & $.066 ; .566$ & .014 \\
\hline $\begin{array}{c}\text { Mujeres/familias identificadas que } \\
\text { viven o han vivido una situación de } \\
\text { VG }\end{array}$ & No & Sí & .200 & $-.037 ; .437$ & .098 \\
\hline $\begin{array}{l}\text { Mujeres/familias identificadas que } \\
\text { viven o han vivido situación de VG }\end{array}$ & No & Sí & .267 & $.043 ; .491$ & .019 \\
\hline $\begin{array}{c}\text { Actividades de prevención de la VG } \\
\text { realizadas en el centro dirigidas al } \\
\text { alumnado }\end{array}$ & No & Sí & -.276 & $-.570 ; .017$ & .065 \\
\hline $\begin{array}{l}\text { Actividades de formación sobre } \mathrm{VG} \\
\text { realizadas por el equipo educativo }\end{array}$ & No & Sí & .260 & $.013 ; .507$ & .040 \\
\hline $\begin{array}{l}\text { Actividades de prevención de la VG } \\
\text { realizadas en el centro dirigidas al } \\
\text { equipo educativo }\end{array}$ & No & Sí & -.186 & $-.439 ; .067$ & .149 \\
\hline
\end{tabular}


Tabla 3

Regresión lineal múltiple para los factores Ayuda especializada, Transversalización de la perspectiva de género y Formación en VG de la escala Medidas.

\begin{tabular}{|c|c|c|c|c|c|}
\hline $\begin{array}{c}\text { Factor Ayuda especializada } \\
\text { Variable }\end{array}$ & $\begin{array}{c}\text { Cat. de } \\
\text { referencia }\end{array}$ & Cat. de riesgo & Beta & IC $95 \%$ & $\mathbf{P}$ \\
\hline $\begin{array}{l}\text { Equipo educativo ha planteado la } \\
\text { necesidad de formación sobre VG }\end{array}$ & No & Sí & .255 & $.032 ; .477$ & .025 \\
\hline Localidad Villa María [VM] & $\begin{array}{l}\text { No es de } \\
\text { VM }\end{array}$ & VM & .214 & $-.075 ; .503$ & .147 \\
\hline $\begin{array}{l}\text { Participación con la comunidad para } \\
\text { articular actividad de prevención y/o } \\
\text { para abordar VG }\end{array}$ & No & Sí & .170 & $-.052 ; .392$ & .133 \\
\hline Nivel & $\begin{array}{c}\text { Otros } \\
\text { niveles }\end{array}$ & Medio & -.177 & $-.418 ; .064$ & .150 \\
\hline \multicolumn{6}{|l|}{$\begin{array}{c}\text { Factor Transversalización de la } \\
\text { perspectiva de género }\end{array}$} \\
\hline Variable & $\begin{array}{c}\text { Cat. de } \\
\text { referencia }\end{array}$ & Cat. de riesgo & Beta & IC $95 \%$ & $\mathbf{P}$ \\
\hline Nivel & $\begin{array}{l}\text { Otros } \\
\text { niveles }\end{array}$ & Primario & -.447 & $-.653 ;-.240$ & .000 \\
\hline $\begin{array}{c}\text { Actividades de prevención de la VG } \\
\text { realizadas en el centro dirigidas al } \\
\text { alumnado }\end{array}$ & No & Sí & .406 & $.165 ; .648$ & .001 \\
\hline $\begin{array}{l}\text { Localidad San José de la Dormida } \\
\text { [SJD] }\end{array}$ & No es SJD & Localidad SJD & -.393 & $-.726 ;-.061$ & .021 \\
\hline Localidad Cruz del Eje [CE] & No es CE & Localidad CE & -.360 & $-.647 ;-.072$ & .014 \\
\hline Años de experiencia en el cargo & & & -.009 & $-.022 ; .003$ & .139 \\
\hline \multicolumn{6}{|l|}{ Factor Formación en $V G$} \\
\hline Variable & $\begin{array}{c}\text { Cat. de } \\
\text { referencia }\end{array}$ & Cat. de riesgo & Beta & IC $95 \%$ & $\mathbf{P}$ \\
\hline $\begin{array}{l}\text { Personal del equipo educativo que } \\
\text { ha vivido o vive una situación de } \mathrm{VG}\end{array}$ & No & Sí & .531 & $.270 ; .792$ & .000 \\
\hline Sexo & Mujer & Varón & -.338 & $-.694 ; .018$ & .062 \\
\hline Localidad VM & No es VM & Localidad VM & .488 & $.191 ; .785$ & .001 \\
\hline Nivel & $\begin{array}{c}\text { Otros } \\
\text { niveles }\end{array}$ & Medio & .294 & $.044 ; .544$ & .022 \\
\hline $\begin{array}{l}\text { Participación con la comunidad para } \\
\text { articular actividad de prevención y/o } \\
\text { para abordar VG }\end{array}$ & No & Sí & -.184 & $-.403 ; .034$ & .098 \\
\hline Zona & Urbana & Rural & -.214 & $-.470 ; .042$ & .100 \\
\hline
\end{tabular}


El análisis de regresión muestra que el factor denominado Vínculos y prejuicios funciona como obstáculo importante para el nivel primario. Para el nivel medio o secundario y para quienes han realizado reuniones con la comunidad para articular actividades de prevención, la escasez de recursos tiene gran relevancia. La importancia otorgada al factor Ayuda especializada como medida posible para prevenir la VG se asocia principalmente a los equipos educativos que han manifestado necesidad de formación en este tema. Quienes han realizado actividades de prevención dirigidas al alumnado y las escuelas de nivel primario valoran más el factor Transversalización de la perspectiva de género como medida. Las escuelas que tienen, dentro de su equipo educativo, a personal que ha vivido o vive una situación de $\mathrm{VG}$ consideran el factor Formación en VG como medida de gran relevancia.

\section{Discusión y conclusiones}

Este trabajo tuvo como objetivo desarrollar y validar las dos escalas en el contexto de la provincia de Córdoba. Por un lado, una escala que permita conocer las opiniones y valoraciones del personal educativo sobre los obstáculos que identifican para afrontar la VG $\mathrm{y}$, por el otro, una escala que posibilite reconocer las medidas que consideran necesarias para abordar la VG. Las escalas validadas son fiables y recogen dimensiones empíricas de las valoraciones sobre obstáculos y medidas en relación con el abordaje de la $\mathrm{VG}$ en las escuelas.

La identificación de obstáculos denominados Vínculos y prejuicios surge con mayor fuerza entre docentes que pertenecen al nivel primario. Esto resultaría coherente, puesto que en este nivel, a diferencia de los otros, suelen existir vínculos más cohesionados entre las familias y la comunidad educativa con una mayor participación en la educación (García Sanz, Hernández Prados, Parra Martínez y Gomariz Vicente, 2016; Gomariz Vicente,
Parra Martínez, García Sanz y Hernández Prados, 2019), lo que permitiría identificar los obstáculos existentes en los núcleos familiares y, a la vez, desarrollar estrategias para modificarlos.

En cuanto a la dimensión vinculada a la escasez de recursos como obstáculo para abordar la VG, solo fue relevante para el nivel medio (secundaria) y para quienes han realizado reuniones con la comunidad para articular actividades de prevención. Es importante considerar que el alumnado que conforma dicho nivel educativo inicia los primeros vínculos amorosos entre pares, a la vez que la exigencia y la dinámica escolar se modifica (Díaz-Aguado y Carvajal, 2011), lo que podría evidenciar, aún más, la importancia de los recursos como "Falta de tiempo para dar todo el temario y tratar estos temas no incluidos en las asignaturas" o "La falta de formación del profesorado para tratar estos temas", por ejemplo ante una situación de VG que ocurra dentro de una pareja en una clase, incluidos en el factor. Asimismo, el haber realizado acciones para articular actividades de prevención ha puesto de relieve la importancia de los recursos para su ejecución. Si bien no se han encontrado estudios que respalden empíricamente dicha asociación, se reconoce la importancia de los recursos para la ejecución de actividades de prevención. Por ejemplo, se ha destacado el rol clave de los recursos para la ejecución, en contextos educativos, de actividades de prevención de consumo de sustancias psicoactivas y violencia en jóvenes (Posada, Moreno y Jaramillo, 2012). Díaz Aguado (2009) también destaca que, a pesar del reconocimiento de la prevención y el rol del sector educativo, los recursos destinados para erradicar la VG en las escuelas son insuficientes.

Al indagar sobre qué medidas fueron valoradas como importantes para responder a esta problemática, se mencionó la necesidad de contar con la ayuda especializada en la escuela que, particularmente, facilite el abordaje de casos, por ejemplo, materiales 
pedagógicos, gabinete psicopedagógico o protocolo de actuación. No es casual que esto haya resultado importante para quienes demandaron mayor formación en el tema, quizás porque son quienes más necesitan conocer los recursos disponibles y apoyarse en ellos. La falta de formación del profesorado en la detección de violencia de género ha sido destacada (Pinedo González, Arroyo González y Berzosa Ramos, 2018).

En relación con otras medidas, como la necesidad de contar con estrategias que contemplen la transversalización de la perspectiva de género, ha sido relevante en particular para las escuelas de nivel primario y para quienes habían realizado actividades de prevención con el alumnado previamente. En este sentido, experiencias previas de actividades llevadas a cabo en la materia (como charlas de sensibilización para docentes 0 familias, o talleres vivenciales con alumnado) permiten identificar la importancia de contar con abordajes pedagógicos desde una mirada que visibilice las desigualdades de género como forma de deconstruir conocimientos y aproximaciones universales (Valls Carol, Torredo Egido, Colás Bravo y Ruiz Eugenio, 2009). Otros elementos característicos del sistema educativo, como métodos tradicionales de enseñanza, poco participativos, expulsivos de la afectividad, o escasamente reflexivos y críticos de las propias prácticas del profesorado han sido señalados como un obstáculo a la hora de implementar la Ley de Educación Sexual Integral (Faur, Gogna y Binstock, 2015; Kornblit et al., 2014). La interiorización desde el inicio de la infancia de comportamientos que reproducen modelos de socialización diferencial entre niños y niñas ha sido ampliamente señalada $\mathrm{y}$, por ello, la importancia de introducir la perspectiva de género en la actividad docente desde los primeros años de educación inicial (Moreno Sánchez y Márquez Vázquez, 2016).

Por último, la necesidad de contar con mayores espacios de formación en estos temas se asocia con aquellas personas que tienen entre sus colegas casos de VG. Se sabe que la formación continua sobre estos temas es crucial para modificar los patrones socioculturales que naturalizan la VG (Barredo Ibáñez et al., 2014; Ruiz Repullo, 2017), por lo que es probable que vivirlo de cerca motive a valorar mejor esta medida. Esta actitud podría encuadrarse en lo que se ha descripto como "espectador de violencia prosocial", es decir, quien como testigo de una situación de VG la enfrenta activamente, por ejemplo, solicitando ayuda (Quintana, Montgomery y Malaver, 2009) o formación en VG. También en contextos universitarios, la formación ha sido una medida muy bien valorada para trabajar en la identificación de las situaciones de VG, especialmente para romper el silencio y desmitificar las situaciones que se normalizan y no se viven como tales (Valls et al., 2009), como pueden ser las que vive el propio profesorado. Este aspecto es importante, especialmente considerando que se ha identificado que mujeres que han sufrido violencia física por parte de una pareja presentaron niveles más altos de sexismo benevolente, caracterizado por actitudes sexistas hacia las mujeres que enfatizan su debilidad y necesidad de protección y las considera de forma estereotipada y limitadas a ciertos roles tradicionales de género (Arbach, Vaiman, Bobbio, Bruera y Lumello, 2019).

Se ha señalado que, en este contexto, tanto para docentes como para personal directivo de las escuelas, la VG es un tema complejo que demanda un mayor nivel de comprensión tanto desde el punto de vista de su conceptualización como de su abordaje (Aizenberg, Saletti-Cuesta y Torres, 2019; Faur, Gogna y Binstock, 2015).

El interés de este estudio reside, en primer lugar, en el aporte al conocimiento sobre la preparación y la actitud hacia la violencia del personal docente, quienes no solo son responsables de mantener y desarrollar un clima de convivencia escolar, sino que son clave en la intervención, prevención (Podestá González, 2019) y ejecución de políticas públicas (Faur, 
Gogna y Binstock, 2015). En esa línea, este trabajo reconoce el rol fundamental de los docentes y el personal directivo en el abordaje de esta problemática. Ejemplo de ello son los ítems que indagan sobre el sexismo por parte del profesorado o la falta de formación sobre estos temas, que apuntan a una mirada crítica sobre el propio rol y contribuyen a la deconstrucción de la violencia desde todos los agentes implicados, incluyendo a docentes, agente fundamental en las complejas dinámicas escolares (Podestá González, 2019).

En segundo lugar, este estudio contribuye a la medición psicométrica de la valoración de obstáculos y posibles medidas para mejorar la igualdad y la prevención de la VG en las escuelas, aspecto poco indagado en el campo de la educación (Faur, Gogna y Binstock, 2015; Tomasini, 2010; Wainerman, Di Virgilio y Chami, 2008).

Como limitaciones del estudio se puede destacar el muestreo no aleatorio que, al no ser representativo de la población estudiada, dificulta la generalización de los resultados. La muestra no probabilística utilizada lleva a considerar los resultados de este estudio como datos iniciales y se sugiere someter la escala a más estudios de validez y confiabilidad.

En segundo lugar, pueden existir dos sesgos vinculados al contexto de recolección de datos. Si bien la mayor parte de los cuestionarios fueron autocumplimentados, en el momento de hacerlo, las personas estaban en grupos y es probable que algunas de ellas hayan consultado a sus pares sobre las respuestas. Por otro lado, algunas personas entregaron los cuestionarios al finalizar la capacitación, con lo cual sus respuestas pueden haberse visto influenciadas por la propia capacitación recibida, y este aspecto no ha sido controlado en los análisis realizados para este trabajo.

También, aunque se trató de una capacitación obligatoria, el hecho de que la última decisión sobre quiénes participaron estuvo tomada por los propios participantes, pudo haber estado influenciado por la identificación de un perfil más afín al tema.
La inclusión de otras variables, como la motivación docente (Muñoz, Lira, Lizama, Valenzuela y Sarlé, 2019) o variables de personalidad del profesorado, como las que incluye el modelo big five, especialmente la afabilidad, es decir la preocupación de tipo altruista y de apoyo emocional a los demás, o la apertura a la experiencia, a ideas o valores nuevos (Hendriks, Hofstee y De Raad, 1999) o la desconexión moral vinculada a la legitimación de la violencia (Martínez González, Robles Haydar, Utria Utria y Amar Amar, 2014) podrían ser valoradas en futuros estudios mediante la inclusión de estas variables y el análisis de sus posibles efectos en las escalas obtenidas en este trabajo.

En conclusión, se validan para el contexto estudiado las dos escalas fiables que permitirían conocer la valoración de obstáculos y medidas para abordar la VG en escuelas públicas, y se constituyen como herramientas factibles para ser utilizadas en este entorno y contribuir al diseño de estrategias y políticas públicas que mejoren la respuesta de este sector ante esta problemática.

\section{Referencias}

Aizenberg, L., Saletti-Cuesta, L. y Torres, E. (2019). Desarrollo de una escala para evaluar opiniones sobre la violencia de género en las escuelas. Revista Argentina de Ciencias del Comportamiento, 11(3), 1-9. https://doi. org/10.32348/1852.4206.v11.n3.24278

Alonso Hernández, C., Cacho Sáez, R., González Ramos, I., Herrera Álvarez, E. y Ramírez García, J. (2016). Guía de buen trato y prevención de la violencia de género: protocolo de actuación en el ámbito educativo. Sevilla: Consejería de Educación, Junta de Andalucía. https://violenciagenero.org/sites/default/files/ guia_buen_trato.pdf

Arbach, K., Vaiman, M., Bobbio, A., Bruera, J. y Lumello, A. (2019). Inventario del sexismo ambivalente: invarianza factorial entre géneros y relación con la violencia de pareja. Interdisciplinaria: Revista de Psicología y Ciencias 
Afines, 36(1), 59-76. https://www.redalyc.org/ jatsRepo/180/18060087004/18060087004.pdf

Barredo Ibáñez, D., Liberia Vayá, I., Macías Cruzatty, A., Palomeque Guillén, V., Delgado Burgos, M. Á., Zurbano-Berenguer, B., ... y Cedeño Delgado, G. (2014). Actitudes y percepciones sobre la violencia de género del personal docente y administrativo y de servicios de Manta: estudio del Colegio 5 de junio. Revista San Gregorio, 1, 38-45. https:// idus.us.es/handle/11441/39084

Bonder, G. (2010). La educación interpelada: Problemáticas emergentes en el campo social y en las relaciones de género. En A. Mingo (Coord.), Desasosiegos. Relaciones de Género en la Educación (pp. 29-54). México DF, México: Plaza y Valdés.

Costello, A. B. y Osborne, J. (2005). Best practices in exploratory factor analysis: four recommendations for getting the most from your analysis. Practical Assessment, Research and Evaluation, 10 (1), 7. https://doi.org/10.7275/ jyj1-4868

Díaz-Aguado, M. J. (2002). Prevenir la violencia contra las mujeres construyendo la igualdad. Programa para educación secundaria. Madrid: Instituto de la Mujer. https://convivencia.files. wordpress.com/2008/11/prevenir_violencia amujeresdiaz-aguado_2002259p.pdf

Díaz-Aguado, M. J. (2006). Convivencia escolary Prevención de la Violencia. Madrid: Ministerio de Educación, Cultura y Deporte. https://www. researchgate.net/publication/299741297 Convivencia_escolar_y_prevencion_de_la_ violencia

Díaz Aguado, M. J. (2009). Prevenir la violencia de género desde la escuela. Revista de Estudios de Juventud, 86, 31-46. https://dialnet.unirioja. es/servlet/articulo? codigo $=3106617$

Díaz-Aguado, M. J. y Carvajal, M. I. (2011). Igualdad y prevención de la violencia de género en la adolescencia. Madrid: Ministerio de Sanidad, Política Social e Igualdad. https:// www.mscbs.gob.es/en/ssi/violenciaGenero/ publicaciones/colecciones/PDFS_COLECCION/libro8_adolescencia.pdf

Faur, E., Gogna, M. y Binstock, G. (2015). La
Educación Sexual Integral en Argentina. Balances y desafios de la implementación de la ley (2008-2015). Buenos Aires: Ministerio de Educación de la Nación.

Flores Bernal, R. (2005). Violencia de género en la escuela: sus efectos en la identidad, en la autoestima y en el proyecto de vida. Revista Iberoamericana de Educación, 38, 67-86. https://doi.org/10.35362/rie380831

García Sanz, M. P., Hernández Prados, M. A., Parra Martínez, J. y Gomariz Vicente, M. A. (2016). Participación familiar en la etapa de educación primaria. Perfiles Educativos, 38(154), 97-117. http://www.scielo.org.mx/scielo. php?pid=S0185-26982016000400006\&scrip$\mathrm{t}=$ sci_arttext

Global Education Monitoring Report Team y United Nations Girls' Education Initiative (2015). La violencia de género relacionada con la escuela impide el logro de la educación de calidad para todos. París: UNESCO. https://en.unesco.org/gem-report/node/818

Gomariz Vicente M. A., Parra Martínez, J., García Sanz, M. P. y Hernández Prados, A. (2019) De lo formal a lo real. Análisis de la participación familiar en asociaciones de madres y padres y consejos escolares. Aula Abierta, 48(1), 85-96. https://doi.org/10.17811/rifie.48.1.2019.85-96

Hendriks, A. A. J., Hofstee, W. K. B. y De Raad, B. (1999). The Five-Factor Personality Inventory (FFPI). Personality and Individual Differences, 27(2), 307-325. https://doi.org/10.1016/ S0191-8869(98)00245-1

Krebs, C., Breiding, M. J., Browne A. y Warner, T. (2011). The association between different types of intimate partner violence experienced by women. Journal of Family Violence, 26, 487-500. https://doi.org/10.1007/s10896-0119383-3

Kornblit, A. (2008). Violencia Escolar y Climas Sociales. Buenos Aires: Biblos.

Kornblit, A. L. F., Sustas, S. E. y Di Leo, P. F. (2014). Género, derechos sexuales, biografía y escuela: articulaciones y tensiones en discursos de docentes de Argentina. Educación y Sociedades, 35(126), 161-178. https://doi. org/10.1590/S0101-73302014000100010 
Martínez González, M. B., Robles Haydar, C., Utria Utria, L. y Amar Amar, J. (2014). Legitimación de la violencia en la infancia: un abordaje desde el enfoque ecológico de Bronfenbrenner. Psicología desde el Caribe, 31(1), 133-160. http://www.scielo.org.co/pdf/psdc/ v31n1/v31n1a07.pdf

Míguez, D. (2009). Tensiones civilizatorias en la conflictividad cotidiana de la dinámica escolar. En C. Kaplan (Comp.) Sociedad y Proceso Civilizatorio (pp. 25-47). Buenos Aires, Argentina: Noveduc.

Ministerio de las Mujeres, Géneros y Diversidad (2020). Plan Nacional de Acción contra las violencias por motivo de género (2020-2022). Buenos Aires: Ministerio de las Mujeres, Géneros y Diversidad. https://www.argentina. gob.ar/sites/default/files/plan_nacional_de_ accion_2020_2022.pdf

Moreno, E. (2000). La transmisión de modelos sexistas en la escuela. En M.A. Santos Guerra (Coord.), El Harén Pedagógico. Perspectiva de Género en la Organización Escolar (pp. 11-31). Madrid, España: Graó.

Moreno Sánchez, E. y Márquez Vázquez, C. (2016). Determinantes implicados en la construcción de la violencia de género. El caso de las escuelas primarias en una provincia de España. Archivos Analíticos de Politicas Educativas, 24(11), 1-24. https://doi. org/10.14507/epaa.24.2132

Morgade, G. (2009). Educación, relaciones de género y sexualidad: caminos recorridos, nudos resistentes. En A. Villa (Comp.), Sexualidad, Relaciones de Género y de Generación: Perspectivas Histórico-Culturales en Educación (pp.19-50). Buenos Aires, Argentina: Novedades Educativas.

Morgade, G. (2011). Toda Educación es Sexual: Hacia una Educación Sexuada Justa. Buenos Aires: La Crujía.

Morgade, G. y Alonso, G. (2008). Cuerpos y Sexualidades en la Escuela. De la Normalidad a la Disidencia. Buenos Aires: Paidós.

Moriana Mateo, G. (2017). Educación en igualdad de género para prevenir la violencia machista. Cuestiones de Género: de la Igualdad y la Dife- rencia, 12, 267-286. https://doi.org/10.18002/ cg.v0i12.4810

Muñoz, C. Lira, B., Lizama, A., Valenzuela, J. y Sarlé, A. (2019). Motivación docente por el uso del juego como dispositivo para el aprendizaje. Interdisciplinaria: Revista de Psicología y Ciencias Afines, 36(2), 233-249. http://repositorio.ucm.cl/handle/ucm/2686

Pinedo González, R., Arroyo González, M. J. y Berzosa Ramos, I. (2018). Género y educación. Detección de situaciones de desigualdad de género en contextos educativos. Contextos Educativos: Revista de Educación, 21, 35-51. https://dialnet.unirioja.es/servlet/articulo?codigo $=6363884$

Podestá González, S. (2019). Metáforas del rol docente en una intervención sobre acoso escolar. Psicoperspectivas, 18(1), 53-63. https://doi.org/10.5027/psicoperspectivas-vol18-issue1-fulltext-1451

Posada, I. C; Moreno, O. y Jaramillo A. C. (2012). Análisis de proyectos gestionados por educadores: el reto de la prevención del consumo de sustancias psicoactivas y violencia en jóvenes en Medellín. Revista Facultad Nacional de Salud Pública, 30(3), 273-281. http://hdl. handle.net/10495/5152

Quintana,A.,Montgomery, W.y Malaver,C.(2009). Modos de afrontamiento y conducta resiliente en adolescentes espectadores de violencia entre pares. Revista de Investigación en Psicología, 12(1), 153-171. http://repositorio.minedu.gob. pe/handle/20.500.12799/2336

Ruiz-Ramírez, R. y Ayala-Carillo, M. (2016). Violencia de Género en instituciones de educación. Ra Ximhai, 12(1), 21-32. https://www. redalyc.org/articulo.oa? $\mathrm{id}=46146696002$

Ruiz Repullo, C. (2017). Estrategias para educar en y para la igualdad: coeducar en los centros. ATLÁNTICAS-Revista Internacional de Estudios Feministas, 2(1), 166-191. https://doi. org/10.17979/arief.2017.2.1.2063

Senado y Cámara de Diputados de la Nación Argentina (2006). Programa Nacional de Educación Sexual Integral. [Ley 26.150]. http://servicios.infoleg.gob.ar/infolegInternet/ anexos/120000-124999/121222/norma.htm 
Senado y Cámara de Diputados de la Nación Argentina (2009). Ley de protección integral para prevenir, sancionar y erradicar la violencia contra las mujeres en Argentina. [Ley 26.485]. https://www.oas.org/dil/esp/ley_de_proteccion_integral_de_mujeres_argentina.pdf

Tomasini, M. (2010). Escuela y construcción de identidades de género: una aproximación a la masculinización de los varones en edad pre-escolar. Revista de Psicología, 19(1), 9-34. https:// doi.org/10.5354/0719-0581.2010.17096

Torres San Miguel, L. (2010). Nuevos retos para la escuela coeducativa: iniciativas y experiencias para la prevención de la violencia de género en las aulas: una mirada general. Tabanque: Revista Pedagógica, 23, 15-44. http://hdl. handle.net/11162/182844

Trucco. D. y Inostroza, P. (2017). Las violencias en el espacio escolar. Naciones Unidas; Santiago de Chile. https://www.cepal.org/es/publicaciones/41068-violencias-espacio-escolar

Valls Carol, R., Torredo Egido, L., Colás Bravo, P. y Ruiz Eugenio, L. (2009). Prevención de la violencia de género en las universidades: valoración de la comunidad universitaria sobre las medidas de atención y prevención. Revista Interuniversitaria de Formación del Profesorado, 23(1), 41-57. https://www.redalyc.org/ pdf/274/27418821004.pdf

Wainerman, C., Di Virgilio, M. y Chami. N. (2008). La escuela y la educación sexual. Buenos Aires: Manantial.

Recibido: 17 de junio de 2020 Aceptado: 7 de octubre de 2021 